\title{
Determinação do campo de tensão efetiva em um problema vetorial elástico-linear via método de elementos finitos
}

\author{
Neves, N. $\mathrm{S}^{1 *}$, Pinheiro, V. $\mathrm{P}^{2}$ \\ 1 PPGEC, Universidade Federal do Espírito Santo, Vitória, ES, Brasil. \\ 2 PPGEM, Universidade Federal do Espírito Santo, Vitória, ES, Brasil.
}

e-mail: natan.sian@gmail.com, vitorpinheiro1987@gmail.com

\section{Resumo}

O presente artigo visa expor os conceitos e a modelagem matemática do método de elementos finitos aplicado a um problema de elasticidade linear de dois graus de liberdade. A discretização do problema é direcionada com base na formulação do método de resíduos ponderados de Galerkin. O problema proposto é composto por uma chapa com um orifício interno sujeita a tração uniforme, sendo realizado uma análise da influência dos elementos do tipo triangulares de 3 nós e quadriláteros de 4, 8 e 9 nós na determinação do campo de tensão efetiva sob critério de Von Mises. Os resultados são comparados com a resposta exata e, em síntese, o método aplicado apresentou um bom desempenho associado a baixos níveis de erro.

Palavras chaves: Elementos Finitos, Elasticidade, Galerkin.

\begin{abstract}
The present article aims to expose the concepts and mathematical modeling of the finite element method applied to a linear elasticity problem of two degrees of freedom. The discretization of the problem is directed based on the Galerkin weighted residue method formulation. The proposed problem is composed of a plate with an internal hole subjected to uniform traction, being carried out an analysis of the influence of triangular type elements of 3 nodes and quadrilaterals of 4, 8 and 9 nodes in the determination of the effective stress field under criterion of Von Mises. In this way, the results are compared with the exact solution of the problem and, in synthesis, the applied method presented a satisfactory performance associated with low levels of error.
\end{abstract}

Keywords: Finite Elements, Elasticity, Galerkin.

\section{Introdução}

A teoria da elasticidade visa modelar o comportamento de sólidos sujeitos aos mais variáveis tipos de solicitações. Mesmo com significativos progressos nas obtenções de inúmeras soluções exatas para problemas, tal tipo de resolução fica restrito a configurações simples e consequentemente a necessidade do desenvolvimento de métodos aproximados [1].

A simulação numérica ganhou espaço no contexto científico e em diversas aplicações práticas relevantes na indústria. Dentre os métodos usados para tais simulações, o método dos elementos finitos (MEF) destacase como técnica amplamente aplicada na solução de uma gama de problemas físicos de naturezas diversas, com um grande avanço específico em problemas da área estrutural.

\section{Formulação do Método de Elementos Finitos}

O método de elementos finitos baseia-se na técnica de resíduos ponderados, onde se busca uma minimização de um resíduo. Primeiramente, por meio dos princípios básicos da mecânica do contínuo é possível escrever equações governantes diferenciais para 
um problema de elasticidade linear bidimensional [1]. Então, previamente, apresentase a equação cinemática ou relação de deformação-deslocamento, como expresso na Equação (1).

$$
\underset{\mathcal{E}}{\underline{\varepsilon}}=\nabla_{s} \underline{\underline{u}}
$$

Onde $\underset{\mathcal{E}}{\mathcal{e}} \underset{\sim}{u}$ são os vetores de deformação específica e deslocamento respectivamente. Em seguida, baseado na conservação de momento linear, considerando um corpo em repouso, a equação de equilíbrio é escrita como posto pela Equação (2).

$$
\nabla_{s}^{T} \underset{\sim}{\sigma}+\underset{\sim}{b}=0
$$

Em que $\underset{\sim}{\sigma}$ e $\underset{\sim}{b}$ são vetores que representam as tensões e forças de campo. A relação constitutiva é direcionada pelo comportamento elástico-linear, conforme a Equação (3).

$$
\sigma=\mathbf{D} \underline{\varepsilon}
$$

Sendo que D é a matriz de propriedades elásticas do material, admitindo o estado plano de tensão. Por fim, devem-se impor condições de contorno, onde pode ser expresso pela soma das parcelas devido os deslocamentos prescritos e as trações na superfície, conforme é posto na Equação (4).

$$
\Gamma=\left\{\begin{array}{l}
\Gamma_{\bar{u}}: \mathrm{u}=\bar{u} \\
\Gamma_{t}: \mathrm{t}=\tau \mathbf{n}
\end{array}\right.
$$

Em que $\mathbf{n}$ é um vetor normal unitário no contorno e $\tau$ é o tensor que contém as tensões planas. A partir da exposição das equações de governo é necessário escreve-las como uma sentença integral. Para tanto, introduz-se uma função auxiliar $w$, com objetivo de reduzir a ordem das derivadas. Tal função pode ser escolhida livremente, desde que seja nula nas fronteiras do tipo essencial [2].

$$
\int_{\Omega}\left(\nabla_{s}^{T} \underset{\sim}{\sigma}+\underset{\sim}{b}\right)^{T} w d \Omega=0
$$

Aplicando a integração por partes, condições de contorno e a equação constitutiva do ma- terial, após manipulações algébricas, chega-se na formulação variacional do problema.

$$
\int_{\Omega}\left(\nabla_{s} w\right)^{T} \mathrm{D} \nabla_{s} \underset{\sim}{u} d \Omega=\int_{\Gamma} w^{T} \mathrm{t} d \Gamma+\int_{\Omega} w^{T} \underset{\sim}{b} d \Omega
$$

Vale destacar que a sentença apresentada na Equação (6) não contém nenhuma aproximação. Como o problema é vetorial, sendo que o campo de deslocamento é $\underline{u}=\left(u_{x}, u_{y}\right)$, necessita-se de uma função de peso do tipo $w=\left(w_{x}, w_{y}\right)$. Então, usando o método de Galerkin é possível escrever os campos na forma discreta.

$$
u=\sum_{j=1}^{n} \mathbf{N}_{\mathrm{j}} u_{j} \quad w=\sum_{i=1}^{n} \mathbf{N}_{\mathrm{i}} \underset{\sim}{w_{i}}
$$

Em que $\mathbf{N}$ é a matriz que contém as funções de forma, onde tais funções podem ser facilmente encontradas na literatura básica [2]. Aplicando as aproximações apresentadas na Equação (7) na Equação (6), chega-se a formulação variacional discreta do problema de elasticidade linear.

$$
\begin{aligned}
& {[\mathbf{K}]\{\mathbf{u}\}=\{\mathbf{F}\}} \\
& \mathbf{K}=\int_{\Omega} \mathbf{B}_{\mathrm{i}}^{T} \mathbf{D} \mathbf{B}_{\mathbf{j}} d \Omega \\
& \mathbf{F}=\int_{\Gamma} \mathbf{N}_{\mathrm{i}}^{T} \mathrm{t} d \Gamma+\int_{\Omega} \mathbf{N}_{\mathrm{i}}^{T} \underset{\sim}{b d} \Omega
\end{aligned}
$$

O sistema algébrico, posto na Equação (8), introduz as matrizes de rigidez $\mathbf{K}$ e o vetor de força externa $\mathbf{F}$. Onde a matriz $\mathbf{B}$ é composta das derivadas das funções de forma em relação ao sistema global. A partir da determinação do campo de deslocamentos, é possível estimar as tensões internas e consecutivamente a tensão efetiva na estrutura, sendo que neste trabalho, considera-se o critério de ruptura de Von Mises [2].

\section{Experimentação Numérica}

O estudo de caso é constituído por uma por uma chapa de $H=10, L=18, r=5$ sob ação de uma tração uniforme $p=100$, conforme Figura 1. Considere $E=72 \mathrm{E} 9, v=0$ 

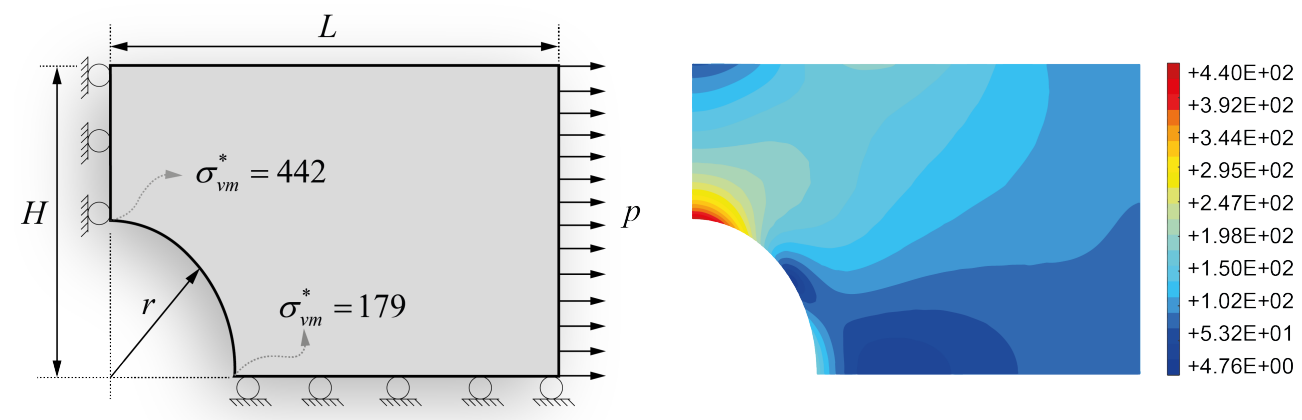

Figura 1: Dimensões da placa sujeita a tração uniforme; Campo bidimensional de tensão efetiva

e espessura unitária. A Tabela 1 apresenta de forma quantitativa os valores das tensões de ruptura nas duas quinas do orifício da estrutura, com seus valores exatos sendo 442 e 179 [3]. Desta maneira, é possível avaliar o desempenho de cada elemento. Realiza-se três simulações para cada tipo de elemento finito, respectivamente com 5, 10 e 15 nós em cada aresta da estrutura.

Tabela 1: Perfomance para cada tipo de elementos.

\begin{tabular}{|c|c|c|c|c|}
\hline \multirow{3}{*}{ T3 } & $3,18 \mathrm{E}+02$ & $28,05 \%$ & $8,43 \mathrm{E}+01$ & $52,91 \%$ \\
\hline & $3,83 \mathrm{E}+02$ & $13,00 \%$ & $1,58 \mathrm{E}+02$ & $11,73 \%$ \\
\hline & $4,27 \mathrm{E}+02$ & $3,40 \%$ & $1,69 \mathrm{E}+02$ & $5,59 \%$ \\
\hline \multirow{3}{*}{ Q4 } & $3,64 \mathrm{E}+02$ & $17,60 \%$ & $1,10 \mathrm{E}+02$ & $38,55 \%$ \\
\hline & $4,26 \mathrm{E}+02$ & $3,61 \%$ & $1,63 \mathrm{E}+02$ & $8,94 \%$ \\
\hline & $4,36 \mathrm{E}+02$ & $1,35 \%$ & $1,72 \mathrm{E}+02$ & $3,91 \%$ \\
\hline \multirow{3}{*}{ Q8 } & $4,20 \mathrm{E}+02$ & $4,98 \%$ & $1,72 \mathrm{E}+02$ & $3,91 \%$ \\
\hline & $4,37 \mathrm{E}+02$ & $1,13 \%$ & $1,67 \mathrm{E}+02$ & $6,70 \%$ \\
\hline & $4,41 E+02$ & $0,23 \%$ & $1,75 \mathrm{E}+02$ & $2,23 \%$ \\
\hline \multirow{3}{*}{ Q9 } & $4,22 \mathrm{E}+02$ & $4,52 \%$ & $1,74 \mathrm{E}+02$ & $2,79 \%$ \\
\hline & $4,38 \mathrm{E}+02$ & $1,02 \%$ & $1,75 \mathrm{E}+02$ & $2,23 \%$ \\
\hline & $4,41 \mathrm{E}+02$ & $0,18 \%$ & $1,76 \mathrm{E}+02$ & $1,79 \%$ \\
\hline Ex: & $4,42 \mathrm{E}+02$ & & $1,79 \mathrm{E}+02$ & \\
\hline
\end{tabular}

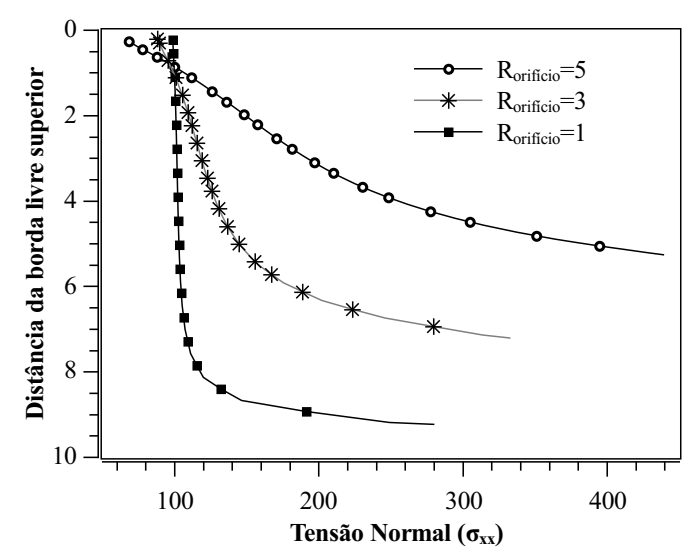

Figura 2: Variação da tensão na direção x para o furo circular central

Em adicional, neste caso, ocorre uma concentração de tensão no ponto superior do orifício. Para avaliar tal comportamento, a Figura 2 ilustra o perfil de tensão normal que atua da face superior até o centro do orifício. Observe que quanto menor o raio do orifício da chapa, a tensão torna-se praticamente constante ao longo da distância, tendo um crescimento rapidamente próximo à borda do orifício.

\section{Considerações Finais}

Os resultados da análise numérica para o caso estudado mostram-se favoráveis no que tange a eficiência do método para o modelo matemático exposto. Nota-se que a escolha do tipo de elemento finito tem influência direta nos resultados obtidos, onde elementos com maior quantidade de nós apresentam melhores resultados, contudo, são elementos demandam um maior número de funções de forma, apresentando uma formulação mais extensa e consecutivamente um maior custo computacional. Em síntese, o elemento quadrilátero de 9 nós foi o que apresentou a melhor reposta, conduzidas por uma boa relação entre refinamento versus precisão.

\section{Referências}

[1] TIMOSHENKO, S. P.; GOODIER, J. N. Teoria da elasticidade, 3ed. Guanabara Dois. 1980.

[2] REDDY, J. N. An introduction to the finite element method. New York, 1993.

[3] PAYEN, D. J; BATHE, K.J. The use of nodal point forces to improve element stresses. Computers \& Structures, v. 89, n. 5-6, p. 485-495, 2011. 